$62^{\text {ème }}$ Congrès de la SFCO, 02007 (2014)

DOI: $10.1051 /$ sfco/20146202007

(C) Owned by the authors, published by EDP Sciences, 2014

\title{
Etude prospective de la prise en charge bucco-dentaire de 51 patients atteints d'un cancer des voies aériennes digestives supérieures avant radiothérapie maxillo-faciale.
}

\author{
Anxionnat $\mathbf{C}^{1}$, Fauroux MA', Favre de Thierrens $\mathbf{C}^{1}$, Boisselier $\mathrm{P}^{2}$, Torres $\mathrm{JH}^{1}$ \\ 1 Service d'Odontologie du CHRU, 549, ave Pr JL Viala, 34295, Montpellier cedex 5, FRANCE \\ 2 ICM, Parc Euromédecine, 208, rue des Apothicaires, 34295 Montpellier cedex 5, FRANCE
}

De nos jours, il est admis que la prise en charge des patients atteints de cancers des voies aériennes digestives supérieures (VADS) doit être multidisciplinaire et organisée. Le rôle de l'odontologiste n'est plus à démontrer tant sur le plan du diagnostic que dans la prise en charge bucco-dentaire. L'amélioration du confort buccal, la prévention des caries et des foyers infectieux, la gestion du risque d'ostéoradionécrose, la réhabilitation prothétique post-chirurgicale sont autant de facteurs qui impliquent l'odontologiste dans cette prise en charge multidisciplinaire (HAS, Novembre 2009). En particulier, dans le cadre de la prévention du risque d'ostéoradionécrose, un bilan bucco-dentaire doit être réalisé de manière systématique avant la radiothérapie, ainsi que l'éviction des foyers infectieux (recommandations AFSOS 2010). Le but de cette étude était d'évaluer l'état bucco-dentaire des patients opérés d'un cancer des VADS avant leur séance de radiothérapie, dans le cadre de la consultation dentaire obligatoire et d'évaluer la compatibilité entre le délai disponible et le délai nécessaire à l'éviction de tous les foyers infectieux buccodentaires.

La consultation dentaire s'est déroulée à l'Institut régional de Cancer de Montpellier (ICM) durant une période de 9 mois. 51 patients ont bénéficié d'un examen clinique et radiographique (panoramique dentaire et/ou cone beam). Les variables retenues ont été : l'âge du patient, son sexe, les facteurs de risques, le type de cancer, les dents déjà absentes, la présence de foyers infectieux, les soins parodontaux, les soins conservateurs, les avulsions à pratiquer. Les foyers infectieux bucco-dentaires ont été notifiés selon la classification utilisée dans les recommandations sur la prise en charge des foyers infectieux buccodentaires (SFCO 2012), où ils sont classés selon 4 catégories (risque faible, moyen, élevé et très fort).
Les résultats de cette étude ont montré que sur les 51 patients examinés, 8 patients présentaient des foyers bucco-dentaires à faible risque ; 28 à risque moyen, 15 à risque élevé et aucun à très fort risque. Sur les 51 patients, 35 nécessitaient des avulsions dentaires ; 34 des soins parodontaux et 4 des soins conservateurs. Pour 25 d'entre eux, des réhabilitations prothétiques étaient à envisager a posteriori. Les délais disponibles entre l'intervention d'exérèse tumorale et les premières séances de radiothérapie n'ont pas toujours été compatibles avec les délais impartis à la réalisation des soins dentaires conservateurs et chirurgicaux et à la cicatrisation : pour 14 patients le début de la radiothérapie a dû être différé ; pour 2 patients, les soins dentaires préconisés n'ont pas pu être entrepris.

La forte proportion de patients qui présentaient des foyers infectieux bucco-dentaires avant irradiation maxillo-faciale dans cet échantillon confirme l'importance de la prise en charge bucco-dentaire spécifique. Par ailleurs, il apparait souhaitable d'établir, en accord avec les autres acteurs de santé, des recommandations de bonnes pratiques claires et précises, afin de disposer d'un protocole standardisé définissant le moment le plus opportun de la prise en charge par chacune des spécialités concernées.

ANXIONNAT Céline celine.anx@hotmail.fr

This is an Open Access article distributed under the terms of the Creative Commons Attribution License 4.0, which permits unrestricted use, distribution, and reproduction in any medium, provided the original work is properly cited. 